\title{
Absorption features in the spectra of X-ray bursting neutron stars
}

\author{
T. Rauch ${ }^{1}$, V. Suleimanov ${ }^{1,2}$, and K. Werner ${ }^{1}$ \\ 1 Institute for Astronomy and Astrophysics, Kepler Center for Astro and Particle Physics, Eberhard Karls University, Sand 1, \\ 72076 Tübingen, Germany \\ e-mail: rauch@astro.uni-tuebingen.de \\ 2 Kazan State University, Kremlevskaja Str. 18, Kazan 420008, Russia
}

Received 5 May 2008 / Accepted 22 August 2008

\begin{abstract}
Context. The discovery of photospheric absorption lines in XMM-Newton spectra of the X-ray bursting neutron star in EXO $0748-676$ by Cottam and collaborators allows us to constrain the neutron star mass-radius ratio from the measured gravitational redshift. A radius of $R=9-12 \mathrm{~km}$ for a plausible mass range of $M=1.4-1.8 M_{\odot}$ was derived by these authors.

Aims. It has been claimed that the absorption features stem from gravitationally redshifted $(z=0.35) n=2-3$ lines of $\mathrm{H}-$ and $\mathrm{He}-\mathrm{like}$ iron. We investigate this identification and search for alternatives.

Methods. We compute LTE and non-LTE neutron-star model atmospheres and detailed synthetic spectra for a wide range of effective temperatures $\left(T_{\text {eff }}=1-20 \mathrm{MK}\right)$ and different chemical compositions.

Results. We are unable to confirm the identification of the absorption features in the X-ray spectrum of EXO $0748-676$ as $n=2-3$ lines of H- and He-like iron (Fe XXVI and Fe XXV). These are subordinate lines that are predicted by our models to be too weak at

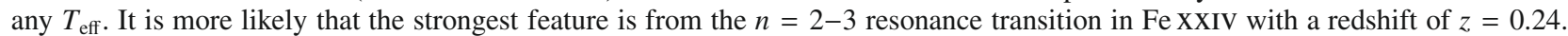
Adopting this value yields a larger neutron star radius, namely $R=12-15 \mathrm{~km}$ for the mass range $M=1.4-1.8 M_{\odot}$, favoring a stiff equation-of-state and excluding mass-radius relations based on exotic matter. Combined with an estimate of the stellar radius $R>12.5 \mathrm{~km}$ from the work of Özel and collaborators, the $z=0.24$ value provides a minimum neutron-star mass of $M>1.48 M_{\odot}$, instead of $M>1.9 M_{\odot}$, when assuming $z=0.35$.

Conclusions. The current state of line identifications in the neutron star of EXO 0748-676 must be regarded as highly uncertain. Our model atmospheres show that lines other than those previously thought must be associated with the observed absorption features.
\end{abstract}

Key words. line: formation - line: identification - scattering - stars: individual: EXO 0748-676 - stars: neutron - X-rays: stars

\section{Introduction}

Cottam et al. (2002, hereafter CPM02) identified discrete absorption features corresponding to electronic transitions in highly ionized iron in the burst spectra of the neutron star in EXO 0748-676 observed with XMM-Newton. They identified $n=2-3$ absorption features of H-like (Fe XXVI) and He-like iron (Fe XXV) in early and late phases of the bursts, respectively. These features provided a redshift measurement of $z=0.35$, corresponding to a mass-radius ratio of $M / R=0.152 M_{\odot} \mathrm{km}^{-1}$. Using this redshift and an estimate for the stellar radius of $R>$ $13.8 \mathrm{~km}$, Özel (2006) inferred a neutron star mass of $M>$ $2.10 M_{\odot}$. However, using Özel's numbers and formulae, we obtain slightly different values $\left(R>12.5 \mathrm{~km}, M>1.9 M_{\odot}\right)$.

The identification of only a few observed lines in burst spectra of NS with unknown redshift is potentially ambiguous. It is the aim of our paper to confirm the proposed line identifications in EXO 0748-676. To this end, we performed LTE and non-LTE model-atmosphere calculations in a wide parameter range using two independently developed stellar atmosphere modeling codes. In this systematic study, we elaborate on our earlier suspicion that an alternative line identification is more likely (Werner et al. 2007).

In the past two decades, spectral analysis of hot, compact stars by means of fully line-blanketed NLTE model atmospheres (e.g. Rauch 2003) has achieved a high level of sophistication. For our analyses, the Tübingen NLTE Model Atmosphere
Package (TMAP ${ }^{1}$, Werner et al. 2003; Rauch \& Deetjen 2003) was used to calculate plane-parallel NLTE model atmospheres that are in radiative and hydrostatic equilibrium. Such model atmospheres were used successfully in the analysis of hot white dwarfs, e.g. LS V $+46^{\circ} 21\left(T_{\text {eff }}=95 \mathrm{kK}\right.$, Rauch et al. 2007) and KPD $0005+5106\left(T_{\text {eff }}=200 \mathrm{kK}\right.$, Werner et al. 2008). TMAP models were also calculated for the extremely hot super-soft X-ray source V $4743 \operatorname{Sgr}\left(T_{\text {eff }}=610 \mathrm{kK}\right.$, Rauch et al. 2005).

The TMAP NLTE model atmospheres can also be employed in the analysis of neutron stars with low magnetic fields, i.e. in the range where the magnetic field strength has no significant impact on atomic data $\left(B \lesssim 10^{12} \mathrm{G}\right)$. Since magnetic fields in low-mass X-ray binaries (LMXBs) are believed to be small, Xray spectra of the neutron star in EXO 0748-676 can be compared with our synthetic spectra.

We calculated TMAP models for the relevant $T_{\text {eff }}$ range and investigated their $T_{\text {eff }}$-dependence (Sect. 2). We describe results of a comparison of LTE and NLTE model-atmosphere fluxes in Sect. 3. A comparison of EXO 0748-676 X-ray observations with our models follows in Sect. 4 and we conclude in Sect. 5.

\section{Model atmospheres and atomic data}

The TMAP code was not especially designed specifically for calculating the burst spectra of neutron stars in LMXBs. TMAP

${ }^{1}$ http://astro.uni-tuebingen.de/ rauch/TMAP/TMAP.html 
Table 1. Statistics of model atoms used in the calculation of our TMAP models. (N)L is the number of levels treated in (N)LTE, RBB (radiative bound-bound) is the number of line transitions. $\mathrm{H}, \mathrm{He}, \mathrm{C}, \mathrm{N}$, and $\mathrm{O}$ are the same for all $T_{\text {eff }}$, while the Fe ionization stages, which are considered, are adjusted, respectively (Table 2).

\begin{tabular}{lccclccc}
\hline \hline Ion & NL & L & RBB & Fe ion & NL & L & RBB \\
\hline H I & 10 & 6 & 45 & Fe XII & 5 & 1 & 3 \\
H II & 1 & - & - & Fe XIII & 7 & 4 & 3 \\
He II & 16 & 18 & 95 & Fe XIV & 8 & 4 & 8 \\
He III & 1 & - & - & Fe XV & 7 & 6 & 5 \\
C V & 29 & 21 & 60 & Fe XVI & 7 & 5 & 10 \\
C VI & 21 & 15 & 49 & Fe XVII & 6 & 8 & 4 \\
C VII & 1 & - & - & Fe XVIII & 4 & 4 & 2 \\
N VI & 17 & 36 & 33 & Fe XIX & 5 & 1 & 3 \\
N VII & 21 & 34 & 55 & Fe XX & 6 & 2 & 4 \\
N VIII & 1 & - & - & Fe XXI & 9 & 4 & 6 \\
O VII & 19 & 16 & 33 & Fe XXII & 6 & 3 & 4 \\
O VIII & 15 & 30 & 30 & Fe XXIII & 7 & 8 & 2 \\
O IX & 1 & - & - & Fe XXIV & 12 & 15 & 28 \\
& & & & Fe XXV & 23 & 30 & 59 \\
& & & & Fe XXVI & 15 & 40 & 30 \\
& & & & Fe XXVII & 1 & - & - \\
\hline
\end{tabular}

Table 2. Fe ionization stages that are considered at individual $T_{\text {eff }}$ (cf. Table 1 and Fig. 1).

\begin{tabular}{crrrrr}
\hline \hline$T_{\text {eff }} / \mathrm{MK}$ & \multicolumn{2}{c}{ ionization stages } & \multicolumn{2}{c}{$T_{\text {eff }} / \mathrm{MK}$} & \multicolumn{2}{c}{ ionization stages } \\
\hline 1 & XII - & XXI & 6 & XXI - & XXVII \\
2 & XVI - & XXV & 7 & XXII - & XXVII \\
3 & XVII - & XXVI & $8-13$ & XXIII - & XXVII \\
4 & XIX - & XXVI & $14-20$ & XXIV - & XXVII \\
5 & XX - & XXVI & & & \\
\hline
\end{tabular}

does not consider general relativistic effects on the radiation transfer within the atmosphere, or velocity fields. Nevertheless, we believe that TMAP models are well suited for our purpose, which is line identification in observed spectra. Comptonization effects are also neglected because Suleimanov \& Werner (2007) demonstrated that at $T_{\text {eff }}=3 \mathrm{MK}$ and $\log g=14.3$ its impact on the emergent spectrum is detectable only at energies higher that about $10 \mathrm{keV}(\lesssim 1.24 \AA)$. It is clear that Comptonization is more important for models with higher $T_{\text {eff }}$ and that it determines the temperature structures and emergent spectra of the hottest models ( $T_{\text {eff }} \gtrsim 20 \mathrm{MK}$, see Lapidus et al. 1986; London at al. 1986; Madej 1991; Madej et al. 2004). The influence of Comptonization is discussed in detail in the next section.

Throughout the paper, we fix the surface gravity in all models to be $\log g=14.39\left(\mathrm{~cm} / \mathrm{s}^{2}\right)$ representing a neutron star with $M=$ $1.4 M_{\odot}$ and $R=10 \mathrm{~km}$.

The model atoms used in our NLTE calculations are summarized in Table 1. From test calculations, we determined the dominant ionization stages and adjusted the model atoms (Table 2) to avoid numerical instabilities due to extremely depopulated ionisation stages. Figure 1 displays the atmospheric structures for models with different $T_{\text {eff }}$. For all elements, we consider level dissolution (pressure ionization) by following Hummer \& Mihalas (1988) and Hubeny et al. (1994). In the latter paper, the Hummer \& Mihalas (1988) method was also considered for the hydrogen atom, but it can be used for other ions as well. The extended opacity tables were indeed calculated in the framework of the Opacity Project (Badnell et al. 2005) up to temperatures of $\sim 10^{7} \mathrm{~K}$ and densities of $\sim 10^{2} \mathrm{~g} \mathrm{~cm}^{-3}$. These opacities agree well with the opacities calculated by the OPAL project

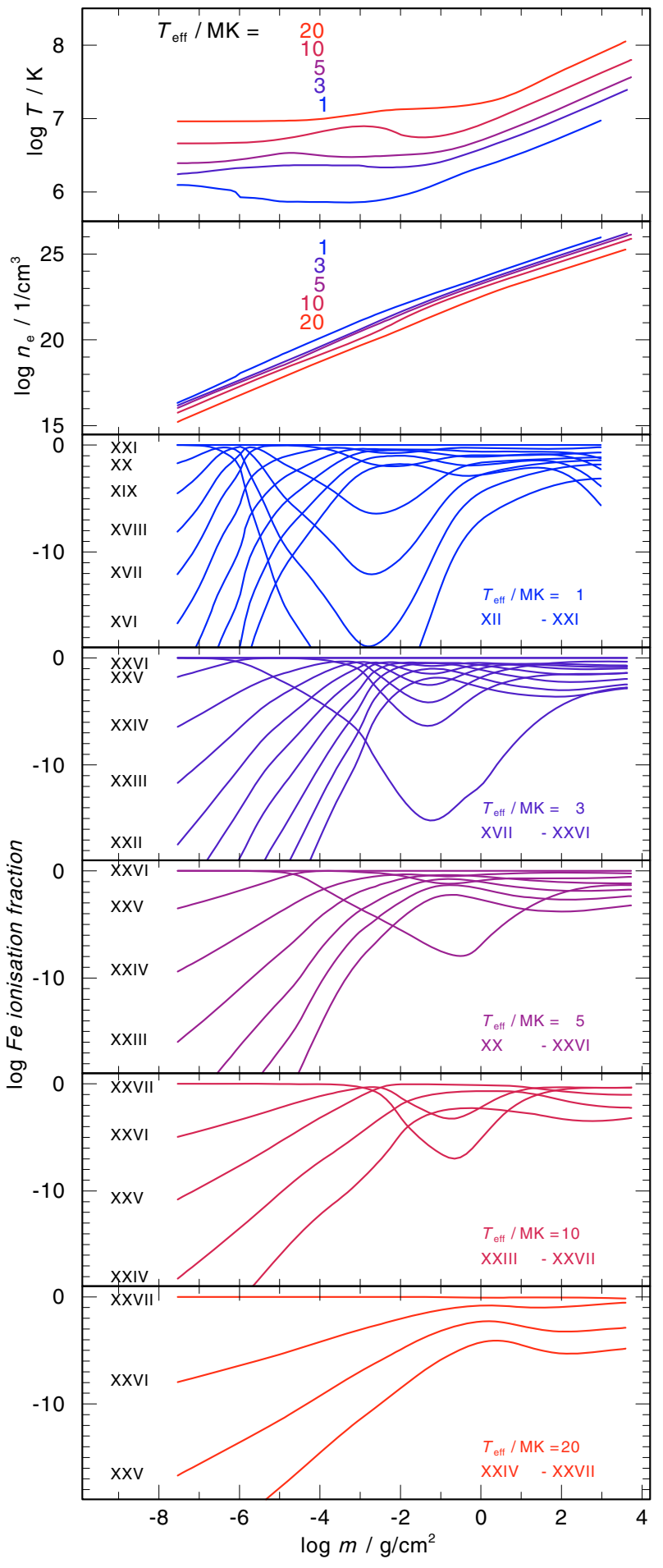

Fig. 1. Atmosphere structure (temperature: 1st panel from top, electron density: 2nd) of our NLTE models which consider opacities of $\mathrm{H}, \mathrm{He}$, $\mathrm{C}, \mathrm{N}, \mathrm{O}$, and Fe with solar abundances, $T_{\mathrm{eff}}=1,3,5,10,20 \mathrm{MK}$. In panels 3 to 7 , the ionization fractions of the iron ions which are used in the calculations are shown. Depending on $T_{\text {eff }}$, the considered ions are selected to well represent the dominant ionization stages in the lineforming region.

(Rogers \& Iglesias 1992). In the OPAL project, the other "physical method" for the occupation densities calculation is used. The emergent spectra in our models are formed at densities of $\leq 10 \mathrm{~g} \mathrm{~cm}^{-3}$, therefore our calculations of the occupation densities are correct. Because of the high particle density in the 


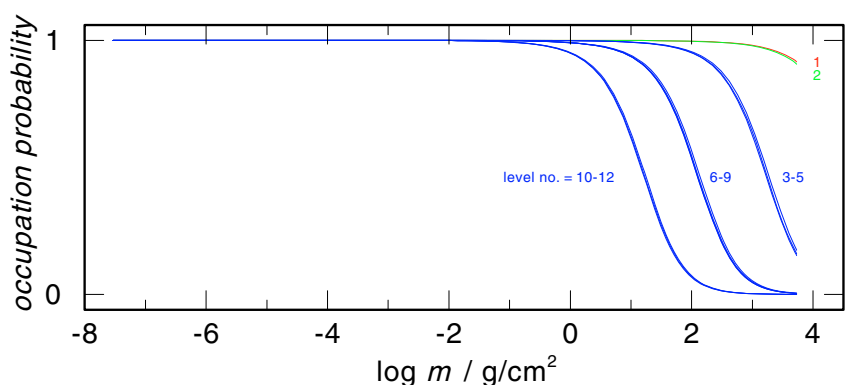

Fig. 2. Occupation probabilities of the Fe XXIV levels (labeled consecutively with increasing excitation energy) in our NLTE model atmosphere with $T_{\text {eff }}=10 \mathrm{MK}$ and solar abundances.

neutron star atmospheres, this is of course an important point for the correct computation of the atmospheric structure in deeper layers. As an example, we display the occupation probabilities of the energy levels of Fe XXIV in a particular model (Fig. 2). Level dissolution (i.e. low occupation probability) is significant for all atomic levels.

The model atoms are constructed using energy levels from NIST $^{2}$ (National Institute of Standards and Technology) and oscillator strengths and photoionization cross-sections calculated by the Opacity Project (TIPTOPbase ${ }^{3}$ ). The complete set of model ions used is available from TMAD, the Tübingen ModelAtom Database ${ }^{4}$.

We compute plane-parallel non-LTE model atmospheres in hydrostatic and radiative equilibrium. Our calculations start from grey model atmospheres that are calculated by TMAP for the range of parameters $-8.0 \leq \log \tau_{\text {Ross }} \leq+2.6$. The atmospheres are represented by 90 depth points, set up equidistantly in $\log \tau_{\text {Ross }}$ between points 1 and 85 (outside to inside), decrease logarithmically by a factor of two from point to point towards the inner boundary. The subsequent non-LTE modeling is performed after transforming from the $\tau_{\text {Ross }}$ on a column-mass scale $m$.

Line broadening due to the Stark effect is considered. According to Cowley (1971), the broadening due to the quadratic Stark effect is approximately given by:

$\gamma_{\text {Stark }}=5.5 \times 10^{-5} \frac{n_{\mathrm{e}}}{\sqrt{T}}\left[\frac{\left(n_{\mathrm{eff}}^{\mathrm{up}}\right)^{2}}{z+1}\right]^{2}$

where $n_{\mathrm{eff}}^{\text {up }}$ is the effective principal quantum number of the upper level, and $z$ is the effective charge seen by the active electron.

The linear Stark effect (e.g. in the case of Fe XXvI) is considered using an approximate formula (Unsöld 1968; Werner et al. 1991)

$\kappa(\Delta \lambda)=\frac{\pi e^{2}}{m c^{2}} \lambda^{2} f \frac{1}{s_{n}^{*} F_{0}} U\left(\frac{\Delta \lambda}{s_{n}^{*} F_{0}}\right)$

with the electric microfield

$F_{0}=2.61 e\left[\sum_{\text {ions }} z_{i}^{3 / 2} n_{i}\right]^{2 / 3}$

where $U(\beta)$ is given by van Dien (1949). A measure of the width of the Stark pattern is given by

$s_{n}=0.0192 \lambda^{2}\left[n_{\text {up }}\left(n_{\text {up }}-1\right)+n_{\text {low }}\left(n_{\text {low }}-1\right)\right] / Z$.

2 http://physics.nist.gov/PhysRefData/ASD/index.html

3 http://vizier.u-strasbg.fr/topbase/

${ }^{4}$ http://astro.uni-tuebingen.de/ rauch/TMAD/TMAD.html

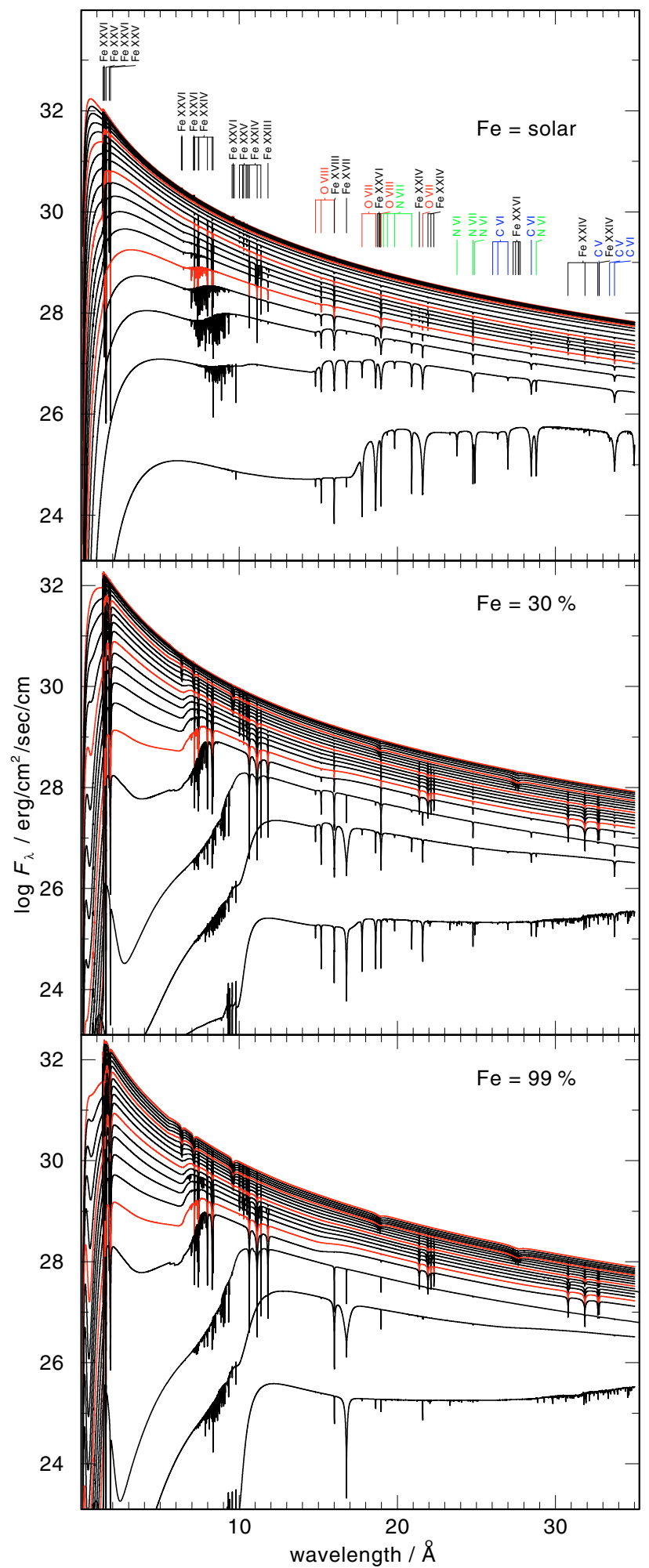

Fig. 3. Astrophysical fluxes of our NLTE models. The three panels display models with different chemical composition (solar, 33\% (by mass), and $99 \%$ Fe content). In each panel models with different effective temperatures are shown ( $T_{\mathrm{eff}}=1-20 \mathrm{MK}$, from bottom to top $)$. Line identifications are given in the top panel.

The dependence of the emergent flux on $T_{\mathrm{eff}}$ is illustrated in Fig. 3. We note that lines of C VI, N VII, and O VIII are detectable in the synthetic spectra up to $T_{\text {eff }} \approx 12 \mathrm{MK}$. Lines of Fe XXIIIFe XXVI visible in the wavelength range from 7 to $12 \AA$ (no 


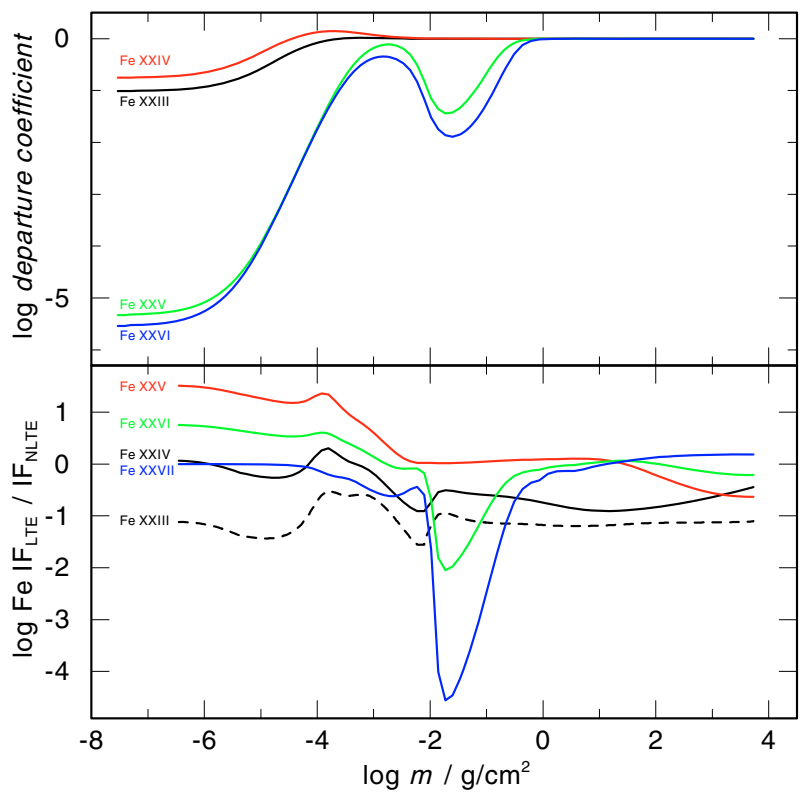

Fig. 4. Upper panel: departure coefficients of the Fe XXIII-XXVI ground states in our $T_{\text {eff }}=10 \mathrm{MK}$ model atmosphere (solar abundances). Bottom panel: ratios of ionisation fractions (IF) of the iron ions for the LTE and NLTE model atmospheres with identical parameters.

gravitational redshift considered) show a strong dependence on $T_{\text {eff }}$ and are therefore very sensitive temperature indicators.

The iron abundance in the photosphere has a significant influence on the emergent spectrum. In Fig. 3, we show fluxes calculated from model atmospheres with solar, 30\%, and 99\% iron abundance (by mass; $\mathrm{H}, \mathrm{He}, \mathrm{C}, \mathrm{N}$, and $\mathrm{O}$ are considered with solar abundance ratios relative to each other).

In the framework of the Virtual Observatory ${ }^{5}(V O)$, all spectral energy distributions (SEDs, $\lambda-F_{\lambda}$ ) from the TMAP model grids described here are available in $V O$ compliant form from the $V O$ service TheoSSA ${ }^{6}$ provided by the German Astrophysical Virtual Observatory $\left(G A V O^{7}\right)$ as well as atables ${ }^{8}$ for the use with $X S P E C^{9}$.

\section{LTE versus NLTE modeling}

For the ground states of iron ions (Fig. 4, upper panel) in a particular model atmosphere $\left(T_{\text {eff }}=10 \mathrm{MK}\right)$, the necessity of considering NLTE effects appears obvious from the LTE departure coefficients, which show the ratio of LTE and NLTE occupation numbers, where the LTE population number is defined relative to the ground state of the subsequent (next highest) ionization stage. However, a closer look into the model structure shows that all atomic levels connected by the line features discussed in the context of EXO 0748-676 have departure coefficients close to unity. The relative deviation from the LTE population density is at most $10 \%$ in the line-forming region. For the $n=2-3$ lines of Fe XXIV-XXVI, this region is confined to the parameter range of $-1.2 \leq \log m \leq+0.2$. As a consequence, NLTE effects on the line profiles are expected to be small. A direct comparison of

\footnotetext{
5 http://www .ivoa.net

6 http://vo.ari.uni-heidelberg.de/ssatr-0.01/

TrSpectra.jsp?

7 http://wwW.g-vo.org

8 http://astro.uni-tuebingen.de/ rauch/TMAF/TMAF.html

9 http://heasarc.gsfc.nasa.gov/docs/xanadu/xspec
}
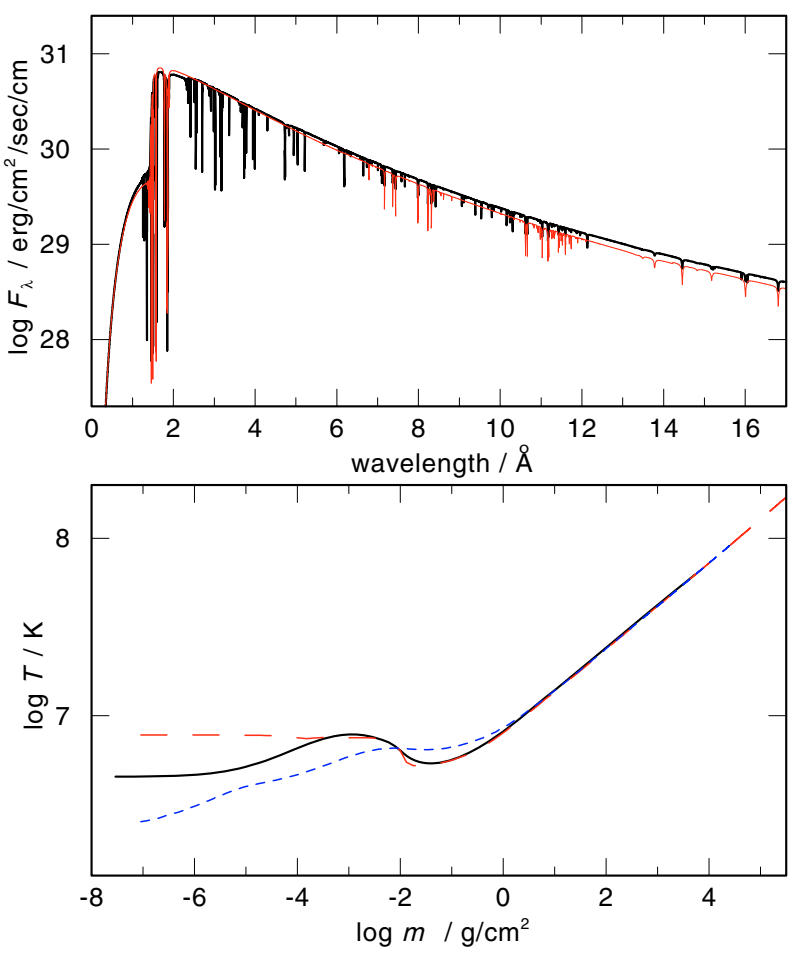

Fig. 5. Top panel: emergent (unredshifted) spectra of LTE models with $T_{\text {eff }}=10 \mathrm{MK}$ and different chemical compositions. Thick line: model of solar composition for all chemical elements up to $Z=30$, thin line: model with $\mathrm{H}, \mathrm{He}, \mathrm{C}, \mathrm{N}, \mathrm{O}, \mathrm{Fe}$ only (solar abundances). Bottom panel: temperature structures of model atmospheres with $T_{\text {eff }}=10 \mathrm{MK}$. Solid lines: NLTE model with $\mathrm{H}, \mathrm{He}, \mathrm{C}, \mathrm{N}, \mathrm{O}, \mathrm{Fe}$ only (solar abundances), long dashes: LTE model with the same chemical composition (note that the high-temperature plateau in this model is caused by Compton scattering), short dashes: LTE model with solar composition for all chemical elements.

the relative densities of the iron ions in LTE and NLTE models confirms this conclusion (Fig. 4, bottom panel; see also below).

For a more detailed comparison, we calculated LTE models (cf. Suleimanov \& Werner 2007; and Ibragimov et al. 2003). In the LTE model-atmosphere code, we are able to model the opacities due to the photoionization of all ions of the 15 most abundant chemical elements, and about 25000 spectral lines of all ions of 26 elements (all elements with atomic number $A \leq 30$, apart from $\mathrm{F}, \mathrm{Sc}, \mathrm{V}$, and $\mathrm{Cu}$ ). Atomic line data were taken from the CHIANTI database (Dere et al. 1997). As for the NLTE atmospheres, we used the occupation-probability formalism for ion population calculations for all considered elements. Compton scattering was also taken into account with this LTE code (Suleimanov \& Poutanen 2006; Suleimanov \& Werner 2007).

A comparison of LTE models with one of the NLTE models is shown in Figs. 5 and 6. We computed three LTE models of the same effective temperature and surface gravity $\left(T_{\text {eff }}=10 \mathrm{MK}\right.$, $\log g=14.39$ ) but with different chemical compositions. The first model had the same chemical composition as the NLTE model ( $\mathrm{H}, \mathrm{He}, \mathrm{C}, \mathrm{N}, \mathrm{O}$, and $\mathrm{Fe}$ all of solar abundances). The second model had solar chemical composition for all considered elements $(A \leq 30)$, and the third model was calculated without hydrogen. It is well known (Lewin et al. 1993) that on a neutronstar surface, the accreted hydrogen can transform into helium by means of pycnonuclear reactions (depending on the accretion rate), and part of the X-ray bursting neutron stars have helium 


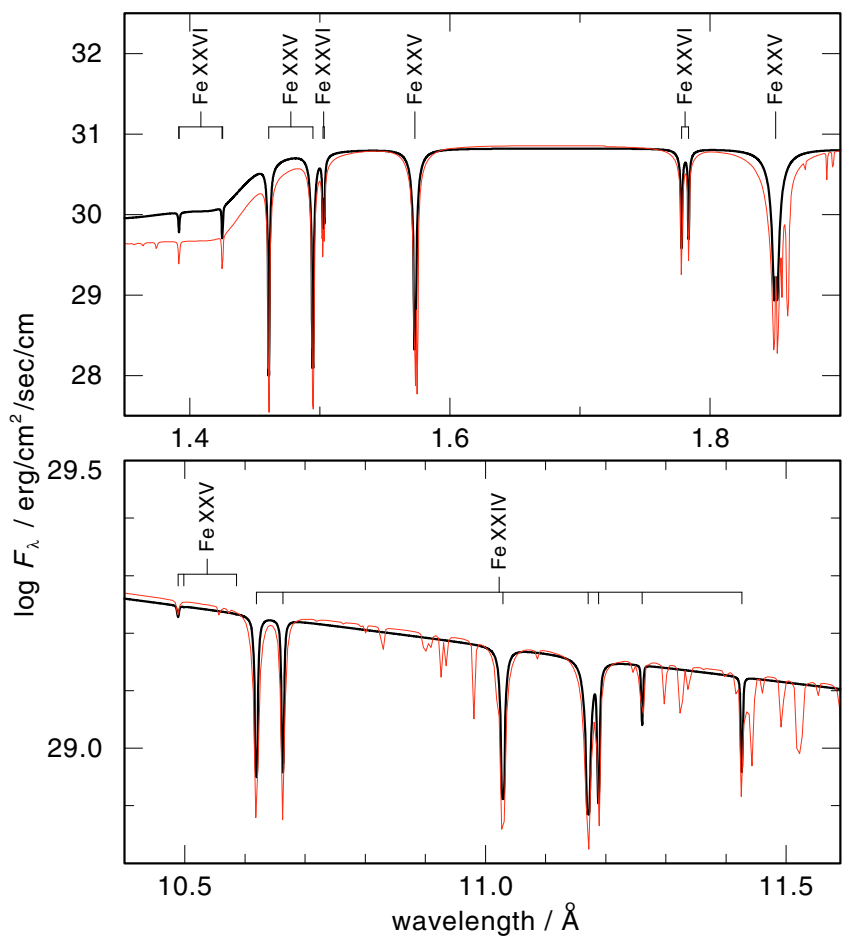

Fig. 6. Comparison of the emergent (unredshifted) spectra of NLTE (thick lines) and LTE models (thin) with $T_{\text {eff }}=10 \mathrm{MK}$ and identical (solar) chemical composition in two different spectral bands. Lines considered in both models are marked.

envelopes without hydrogen. Therefore, we considered a helium model with solar composition of the other chemical elements.

The temperature structure of the first model was similar to the temperature structure of the NLTE model at $\log m>-3$, but the temperature structure of the second model differed in the layers with $\log m<0$, where the emergent flux was formed (Fig. 5, bottom panel). Therefore, the chemical composition of the heavy elements was more important than the NLTE effects. This was also true for spectral energy distribution (Fig. 5, top panel; Fig. 6). This was due to the influence of the large number of other heavy element spectral lines in the 2-8 $\AA$ band (compare spectra in Figs. 3 and 5). The spectrum of the first model was similar to that of the NLTE model than the spectrum of the second model (Figs. 5 and 6). The difference between the emergent spectra of NLTE and LTE model atmospheres with identical chemical composition is significant only at $\lambda<2 \AA$. The spectral line strengths are weaker in the second model because the temperature is higher in the line-formation layers $(-2.6<\log m<+0.4)$ (Fig. 5, bottom panel; Fig. 10).

The helium model atmosphere had lower temperatures at deep layers $(\log m>-2)$ than the second model, and it showed slightly stronger depths of the spectral lines (Figs. 7 and 10). However, the difference between helium and hydrogen models was not significant, and the qualitative conclusions for the hydrogen models were also applicable to the helium models.

LTE model atmospheres of solar composition were also calculated by taking Compton scattering into account. In Fig. 8 , the temperature structures and emergent spectra of these models compared with Thomson-scattering models are shown. It is possible to make the following conclusion from this figure. Comptonization is significant for models with $T_{\text {eff }} \geq 15 \mathrm{MK}$, but the models with lower temperatures and solar chemical composition can be considered without Compton scattering.
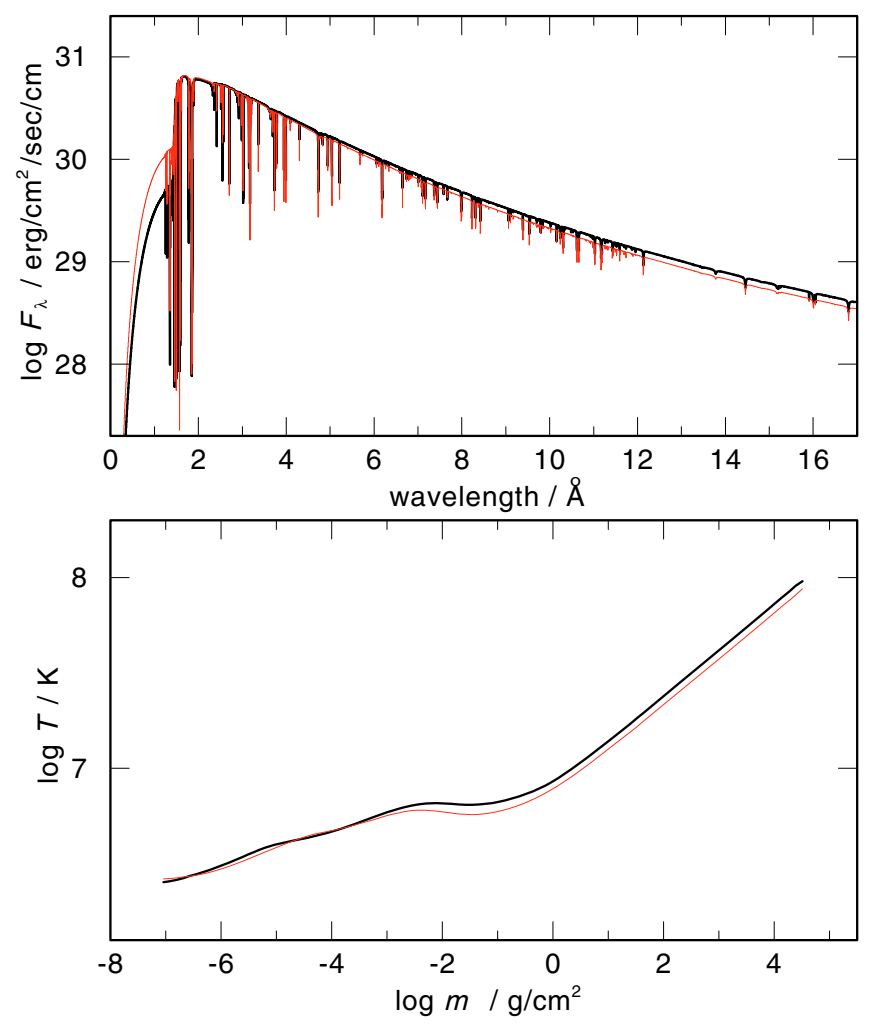

Fig. 7. Emergent (unredshifted) spectra (top panel) and temperature structures (bottom panel) of LTE model atmospheres with $T_{\text {eff }}=10 \mathrm{MK}$ and different chemical compositions. Thick line: model with solar composition for all chemical elements, thin: helium model (without hydrogen) with solar abundance of heavy elements.
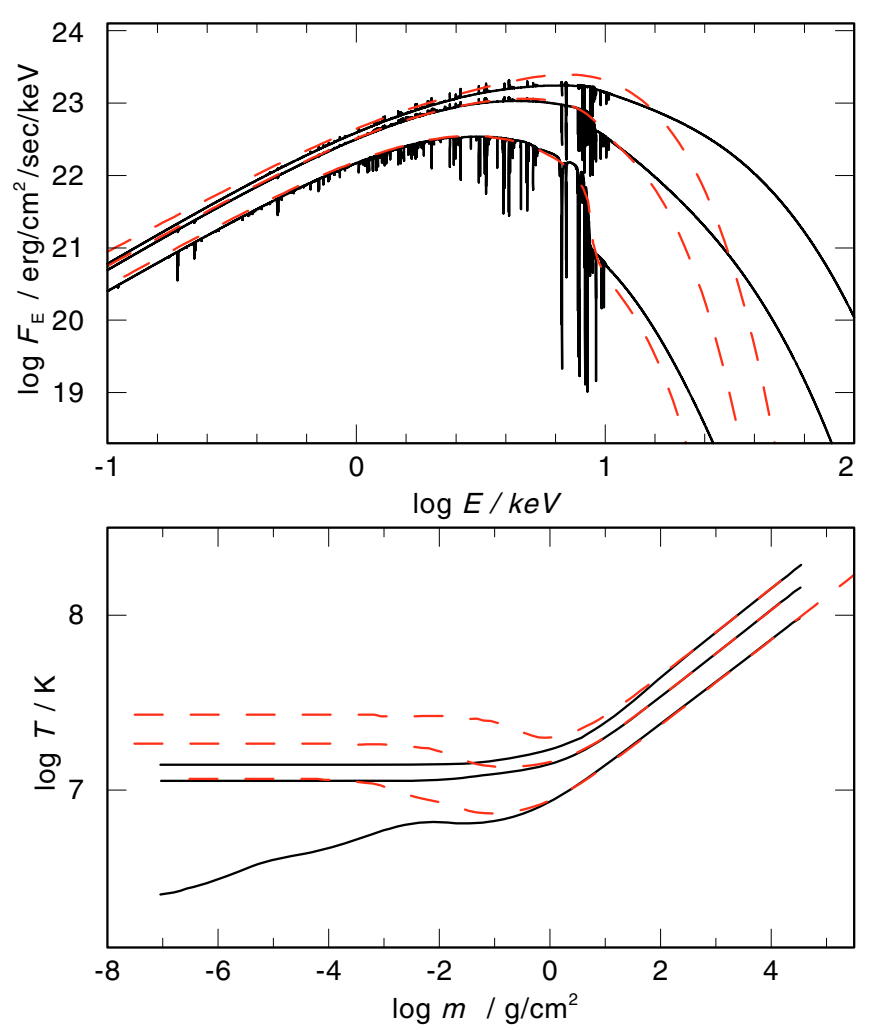

Fig. 8. Emergent (unredshifted) spectra (top panel) and temperature structures (bottom panel) of LTE models with $T_{\text {eff }}=10,15,20 \mathrm{MK}$ for Thomson (solid curves) and Compton scattering (dashed curves). 


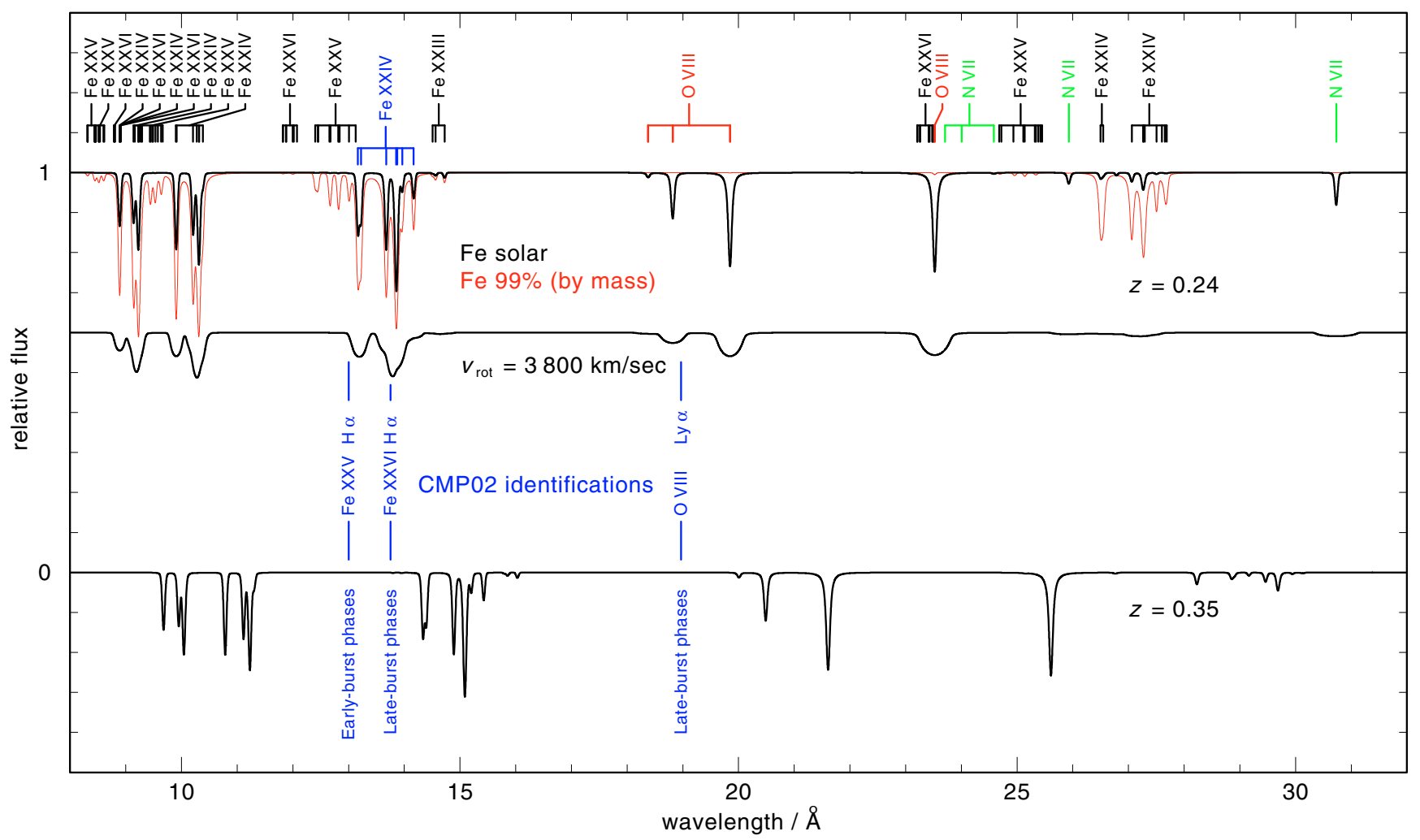

Fig. 9. Comparison of NLTE model-atmosphere fluxes calculated from $T_{\text {eff }}=8 \mathrm{MK}$ models with different iron content. This figure can be directly compared with the observed spectra of EXO 0748-676 presented in Fig. 1 of CPM02 (therefore, all spectra are convolved with a Gaussian of $F W H M=0.124 \AA$, which is about 2.5 times lower in quality than XMM-Newton's RGS spectral resolution). At $z=0.35$ (bottom), there is no fit to the observation. The identification of Fe XXV by CMP02 is unlikely. At a significantly lower redshift of $z=0.24$ (top), the observed line features would originate instead from Fe XXIV.

\section{EXO 0748-676}

CPM02 used blackbody flux distributions to determine the $T_{\text {eff }}$ of the neutron star in EXO 0748-676 in the early- and lateburst phase and derived $k_{\mathrm{B}} T_{\mathrm{eff}} \approx 1.8 \mathrm{keV}\left(T_{\mathrm{eff}} \approx 20.9 \mathrm{MK}\right)$ and $k_{\mathrm{B}} T_{\text {eff }} \leq 1.5 \mathrm{keV}\left(T_{\text {eff }} \leq 17.4 \mathrm{MK}\right)$, respectively. They identified photospheric absorption lines of Fe XXV at a redshift of $z=0.35$. In Figs. 9 and 10, we compare theoretical spectra for the XMM-Newton wavelength range. It is well known (Lapidus et al. 1986; London at al. 1986; Madej 1991; Madej et al. 2004) that the color temperature of an X-ray bursting neutron stars is higher than the effective temperature by a factor of 1.5-1.7; for comparison with late-burst phase observations, we therefore chose models with $T_{\text {eff }} \approx 10 \mathrm{MK}$.

Figure 9 (top) displays spectra from our $T_{\mathrm{eff}}=8 \mathrm{MK}$ models in the wavelength range $8-32 \AA$. It can be compared directly with the observed spectra of EXO 0748-676 shown in Fig. 1 of CPM02. To consider rotational broadening, we assume that $R=$ $13.5 \mathrm{~km}$ (cf. Fig. 13). At $v_{\text {rot }}=45 \mathrm{~Hz}$ (Villarreal \& Strohmayer 2004), this results in an equatorial velocity $v_{\text {rot }}=3800 \mathrm{~km} \mathrm{~s}^{-1}$. The synthetic spectrum in the middle panel of Fig. 9 is convolved with the respective rotational profile (see Chang et al. 2006, for a more detailed treatment of rotational broadening).

The Fe XXV $n=2-3$ absorption features (at rest wavelength $\approx 10.5 \AA$ in Fig. 11) are weak even in the Fe-dominated model and completely absent in the Fe-solar model (features at $\approx 13 \AA$ in Fig. 9 at $z=0.24$ ). Hence, at $z=0.35$ there is no absorption feature at $13 \AA$ that can reproduce the observations. With increasing $T_{\text {eff }}$ the Fe XXV lines become slightly stronger, reaching maximum strength at $T_{\text {eff }}=12 \mathrm{MK}$, although, they remain far weaker than the Fe XXIV $n=2-3$ absorption lines (Fig. 11). The reason is the following. The Fe XXV ionisation stage dominates the considered atmospheres (Fig. 1), but the lines at 10.33, $10.48 \AA$ (for which identifications were suggested by CPM02) correspond to high energy $(E \approx 6.7 \mathrm{keV})$ levels for which the Boltzmann factor $\exp (-E / k T)$ is low. Lines of Fe XXIV originate in ground-level transitions that are more common than the highenergy level transitions of Fe XXV. We remark that occupation probabilities are lower for high-energy levels (see e.g. Fig. 2). Therefore, the high-energy levels of Fe XXV are even less populated than the ground level of Fe XXIV.

Relative flux spectra for LTE models with $T_{\mathrm{eff}}=10 \mathrm{MK}$ are shown in Fig. 10. This figure illustrates the influence of the chemical composition on the emergent flux. Clearly, the above conclusion about line identification is unchanged.

Therefore, from our results it is more likely that the observed features are due to the resonance multiplet $(n=2-3)$ of Lilike iron (Fe XXIV). This would, however, require a significantly lower redshift of $z=0.24$. We briefly discuss the implications of this result for the neutron star parameters.

Figure 13 shows the allowed values for mass and radius in EXO 0748-676 for redshifts $z=0.24$ and $z=0.35$ compared to various theoretical mass-radius relations. These relations represent typical relations for a stiff (BalbN2), moderate (SLy4), soft (BPAL12), and strange matter (SS) equation of state. Details are given in Haensel et al. (2006). While $z=0.35$ corresponds to radii of $R=9-12 \mathrm{~km}$ for a mass-range of $M=1.4-1.8 M_{\odot}$, our redshift $z=0.24$ implies larger radii of $R=12-15 \mathrm{~km}$, which corresponds to stiff equations-of-state and excludes massradius relations based on exotic matter. This result agrees with 


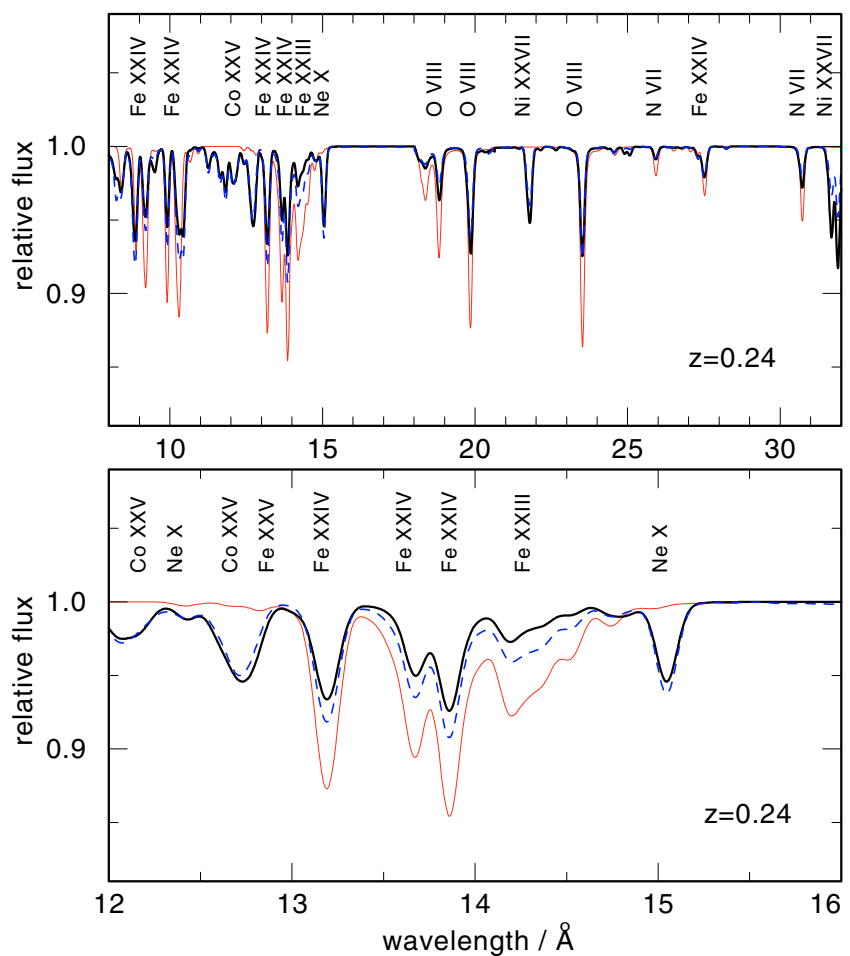

Fig. 10. Emergent redshifted $(z=0.24)$, normalized fluxes of our LTE models with $T_{\text {eff }}=10 \mathrm{MK}$ and different chemical compositions. Thick line: model with solar composition for all chemical elements, dashed: model with $\mathrm{H}, \mathrm{He}, \mathrm{C}, \mathrm{N}, \mathrm{O}, \mathrm{Fe}$ only (solar abundances), thin: helium model. All fluxes have been convolved with a Gaussian $(F W H M=$ $0.124 \AA$ A, see Fig. 9).

the study of EXO 0748-676 by Özel (2006) using additional observational constraints. With $z=0.35$ and using this author's formulae and input values one obtains minimum values of mass and radius, such that $M \geq 1.9 M_{\odot}$ and $R \geq 12.5 \mathrm{~km}$. A reduction in redshift to $z=0.24$ would have a negligible effect on their radius determination but their lower mass limit would be reduced to $M>1.48 M_{\odot}$.

In observations of EXO 0748-676 bursts by Cottam et al. (2008), no significant photospheric line features were detected. Cottam et al. (2008) discussed several possible reasons for this non-detection. They found no conclusive evidence for a different photospheric temperature. Our models indicate that the Fe XxIV lines become weaker towards higher $T_{\text {eff }}$ and disappear at about $T_{\text {eff }}=14 \mathrm{MK}$ (Fig. 11). Considering the rotation of EXO 0748-676, we would expect a featureless spectrum as soon as the temperature exceeds about $T_{\text {eff }}=12 \mathrm{MK}$.

\section{Conclusions}

We have performed model-atmosphere calculations to describe the X-ray spectra of thermal radiation from neutron stars. We have compared our computed spectra with X-ray burst spectra of the LMXB EXO 0748-676. We have been unable to confirm the line identification by CPM02 as being due to subordinate transitions of $\mathrm{H}$ - and He-like iron. These line features were too weak at any $T_{\text {eff }}$ and iron content. Our models suggested that a more likely identification was the resonance line of Li-like iron. As a consequence, the measured line redshift was $z=0.24$ rather than $z=0.35$. This implied a larger neutron star radius of $R=$ $12-15 \mathrm{~km}$ for the mass range $M=1.4-1.8 M_{\odot}$.

We compared results from two entirely different model codes, a LTE and a NLTE code, and concluded that NLTE effects

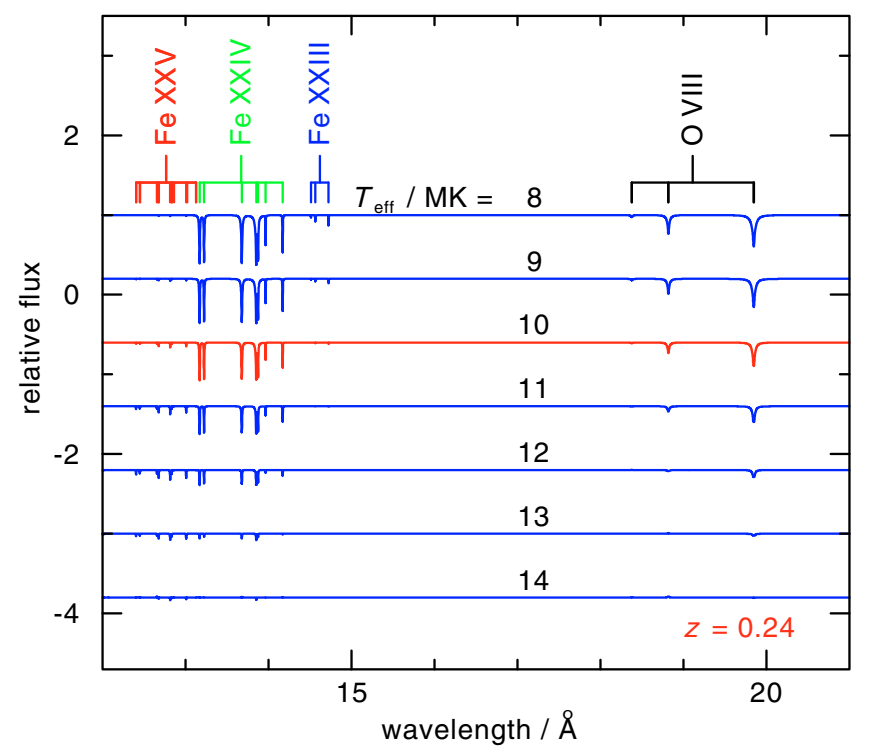

Fig. 11. Comparison of redshifted $(z=0.24)$ NLTE model-atmosphere fluxes calculated from $T_{\text {eff }}=8-14 \mathrm{MK}$ models of solar iron abundance. Note that for clarity these lines have not been convolved with either the instrument's resolution or any rotational profile (cf. Fig. 9).

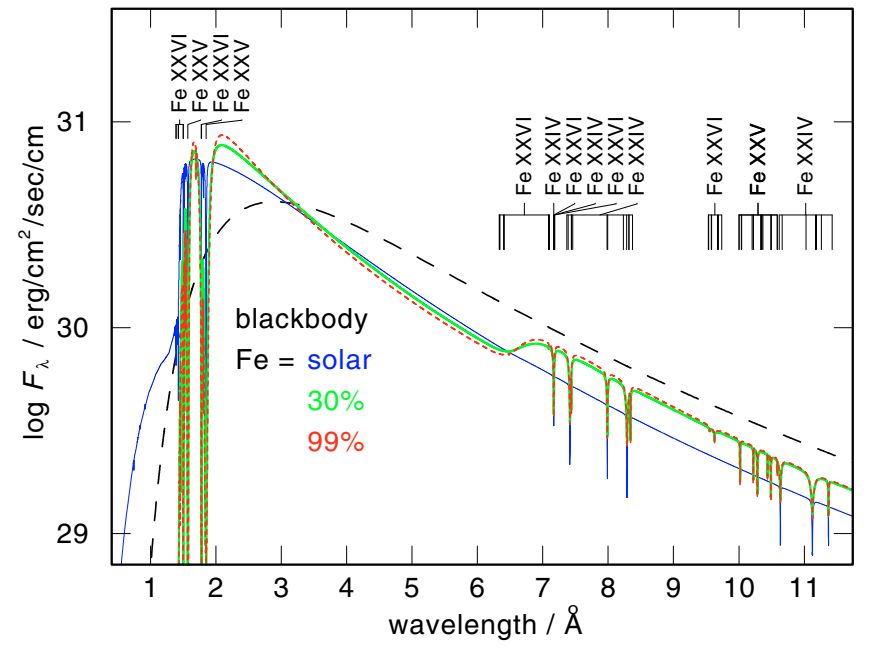

Fig. 12. Comparison of unredshifted NLTE model-atmosphere fluxes calculated from $T_{\text {eff }}=10 \mathrm{MK}$ models with different iron content (thin: solar, thick: $30 \%$ Fe content, short dashes: $9 \%$ Fe). The long-dashed line represents a blackbody spectrum.

were less important than uncertainties in the chemical composition of the bursting neutron-star atmospheres. Given the current state of observations, NLTE effects on continuum shape and spectral line profiles are negligible. On the other hand, we investigated the influence of the various chemical compositions and found that the derived conclusion concerning line identification does not depend on the chemical composition.

We investigated the relevance of Compton electron scattering and found that it was unimportant for solar composition models with $T_{\text {eff }}<15 \mathrm{MK}$.

The comparison of model-atmosphere spectra with blackbody flux distributions (Fig. 12) has shown that modelatmosphere spectra peak at higher energies and have a higher peak intensity. A determination of $T_{\text {eff }}$ by assuming blackbody spectra, as performed by CPM02, therefore overestimates $T_{\mathrm{eff}}$ (cf. Rauch et al. 2005). 


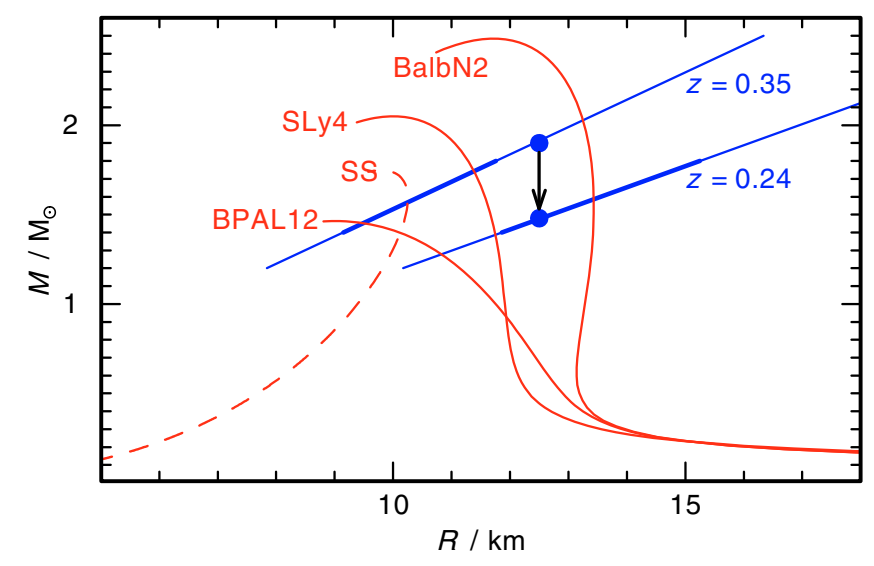

Fig. 13. Allowed values for $M$ and $R$ of EXO 0748-676 for redshifts $z=$ 0.24 and $z=0.35$ (straight lines; thick portions of the graphs denote the mass range $1.4-1.8 M_{\odot}$ ) compared to various theoretical $M-R$ relations (Haensel et al. 2006). The thick dot on the $z=0.35$ line denotes the minimum $M$ and $R$ derived by Özel (2006). The arrow indicates the shift of this result when we assume $z=0.24$. A description of the theoretical mass-radius relations is given in the text.

Acknowledgements. We gratefully thank the referee for helpful comments that improved the final manuscript. We thank Eric Kuulkers for pointing out an error in a previous version for the paper. T.R. is supported by the German Astrophysical Virtual Observatory (GAVO) project of the German Federal Ministry of Education and Research (BMBF) under grant 05 AC6VTB, V.S. by the DFG (grant We 1312/35-1 and SFB/TR7 "Gravitational Wave Astronomy") and partially supported by the Russian President's program for support of leading science schools (grant Nsh-4224.2008.2).

\section{References}

Badnell, N. R., Bautista, M. A., Butler, K., et al. 2005, MNRAS, 360, 458 Chang, P., Bildsten, L., \& Wasserman, I. 2005, ApJ, 629, 998
Chang, P., Morsink, S., Bildsten, L., \& Wasserman, I. 2006, ApJ, 636, L117

Cottam, J., Paerels, F., \& Méndez, M. 2002, Nature, 420, 51 (CPM02)

Cottam, J., Paerels, F., Méndez, M., el al. 2008, ApJ, 672, 504

Cowley, C. R. 1971, The Observatory, 91, 139

Dere, K. P., Landi, E., Mason, H. E., Monsignori Fossi, B. C., \& Young, P. R. 1997, ApJS, 125, 149

Haensel, P., Potekhin, A. Y., \& Yakovlev, D. G. 2006, Neutron Stars 1. Equation of State and Structure (Berlin: Springer)

Hubeny, I., Hummer, D., \& Lanz, T. 1994, A\&A, 282, 151

Hummer D. G., \& Mihalas D. 1988, ApJ, 331, 794

Ibragimov, A. A., Suleimanov, V. F., Vikhlinin, A., \& Sakhibullin, N. A. 2003, Astron. Rep., 47, 186

Lapidus, I. I., Sunyaev, R. A., \& Titarchuk, L. G. 1986, Soviet Ast. Lett., 12, 383 Lewin, W. H. G., van Paradijs, J., \& Taam, R. E. 1993, Space Sci. Rev., 62, 223 London, R. A., Taam, R. E., \& Howard, W. M. 1986, ApJ, 306, 170 Madej, J. 1991, ApJ, 376, 161

Madej, J., Joss, P. C., \& Różańska, A. 2004, ApJ, 602, 904

Molkov, S. V., Grebenev, S. A., \& Lutovinov, A. A. 2000, A\&A, 357, L41

Özel, F. 2006, Nature, 441, 1115

Rauch, T. 2003, A\&A, 403, 709

Rauch, T., \& Deetjen, J. L. 2003, in Stellar Atmosphere Modeling, ed. I. Hubeny, D. Mihalas, \& K. Werner (San Francisco: ASP), ASP Conf. Ser., 288, 103

Rauch, T., Orio M., Gonzales-Riestra, C., \& Still, M. 2005, in 14th European Workshop on White Dwarfs, ed. D. Koester, \& S. Moehler (San Francisco: ASP), ASP Conf. Ser., 334, 423

Rauch, T., Ziegler, M., Werner, K., et al. 2007, A\&A, 470, 317

Rogers, F. J., \& Iglesias, C. A. 1992, A\&AS, 79, 507

Suleimanov, V., \& Poutanen, J. 2006, MNRAS, 369, 2036

Suleimanov, V., \& Werner, K. 2007, A\&A, 466, 661

Unsöld, A. 1968, Physik der Sternatmosphären (Berlin: Springer) van Dien, E. 1949, ApJ, 109, 452

Villarreal, A. R., \& Strohmayer, T. E. 2004, ApJ, 614, L121

Werner, K., Heber, U., \& Hunger, K. 1991, A\&A, 244, 437

Werner, K., Dreizler, S., Deetjen, J. L., et al. 2003, in Stellar Atmosphere Modeling, ed. I. Hubeny, D. Mihalas, \& K. Werner (San Francisco: ASP), ASP Conf. Ser., 288, 31

Werner, K., Nagel, T., Rauch, T., \& Suleimanov, V. 2007, Adv. Space Res., 40, 1512

Werner, K., Rauch, T., \& Kruk, J. W. 2008, A\&A, 474, 591 